\title{
Improving early recognition of delirium using SQiD (Single Question to identify Delirium): a hospital based quality improvement project
}

\author{
Elaine McCleary, Pamela Cumming
}

School of Nursing \& Midwifery, University of Dundee

\begin{abstract}
Delirium is a serious condition associated with poor outcomes which can be prevented and treated if recognised early. Older people and people with dementia or severe illness are more at risk of delirium. SQiD is a simple prompt question which asks, "Is this patient more confused than before?" Focusing specifically on patients aged 75 and over, this project aimed to increase awareness and usage of SQiD to help improve early recognition of delirium, in accordance with the Healthcare Improvement Scotland national initiative. This project was carried out by two student nurses during an eight week clinical placement in the acute surgical receiving unit (ASRU) of Ninewells Hospital, Dundee, Scotland.
\end{abstract}

Qualitative and quantitative methodology was used to establish baseline data which revealed that only $35 \%$ of the multidisciplinary team (MDT) were aware of SQiD, with only $15 \%$ using SQiD. Initial activities involved raising awareness of SQiD by means of information cards and posters. Once awareness was raised, the usage of the SQiD question by nurses was tested. Finally, the SQiD question was incorporated into the nursing care round forms and usage recorded. Following these awareness raising activities we noted an increase of $83 \%$ awareness and $20 \%$ use of SQiD. Incorporating the SQiD question into the hourly care round forms increased awareness to $100 \%$ and usage to $50 \%$.

Although this small scale project could be viewed as a success, the requirements for sustainability depend upon further implementation and spreading of the change. Sustained improvement is also dependent upon the implementation of the care rounds.

As nursing students, undertaking this improvement project has provided valuable lessons in both quality improvement science and personal learning. The improved knowledge and understanding of effective communication and the intricacies of team working is transferrable and can be applied to future nursing practice.

\section{Problem}

Older people and people with dementia, severe illness, or a hip fracture are more at risk of delirium. People who develop delirium may spend longer in hospital and therefore have increased risk of hospital acquired complications such as infection, falls, and pressure sores. They also have increased risk of developing dementia, are more likely to require long-term care and are more likely to die.[1] A local audit of medical notes revealed that recognition of delirium required improvement within the acute surgical receiving unit (ASRU) of Ninewells Hospital, Dundee, Scotland.

\section{Background}

Understanding of delirium is poor yet it is reported to affect up to $56 \%$ of hospital inpatients.[2] In association with the Older People in Acute Care Collaborative (OPACC), Healthcare Improvement Scotland (HIS) aims to improve the identification and immediate management of delirium in people aged 75 and over in acute care. Ultimately, early recognition improves patient safety and reduces the burden on hospital resources.[1]

Single question to identify delirium (SQiD) is a simple prompt question which asks, "Is this patient more confused than before?" and was first introduced to multi disciplinary staff during delirium training sessions. Asking the SQiD question on a regular basis can identify changes in a patient's condition, which could potentially be delirium. Recognising that a person is more confused than before should trigger escalation to medical staff and initiation of 4AT rapid assessment test for delirium. A previous study compared the efficacy of SQiD with delirium assessments such as the confusion assessment method (CAM) and has suggested the simplicity of SQiD as a single item tool makes it more likely to be used by staff and incorporated in to history taking practice.[3] Its simplicity was appealing as this project was initially aimed at the multi-disciplinary team (MDT) which is comprised of non clinical staff.

Care round forms are a means of documenting basic care such as comfort, environment, plan and elimination needs of patients and are designed to be completed by nursing staff each hour during the day and two hourly overnight. With the recent introduction of the care rounds to the ASRU, an opportunity presented itself to incorporate SQiD into the care round forms that provided staff with an opportunity to identify changes in patients cognitive function and subsequently recognise delirium earlier.

\section{Baseline measurement}


A questionnaire, designed to gather qualitative and quantitative data, was distributed to the MDT to establish current awareness and use of SQiD within ASRU. The MDT consisted of medical, nursing, ancillary, clerical, and allied health professionals, with 20 completed questionnaires (45\%) being returned within a two week period. This revealed a baseline of 35\% staff awareness with 15\% using SQiD. Subsequently, it was decided to narrow the scope of the project to focus specifically on nursing staff due to the limited eight week timescale of the clinical placement.

In an effort to improve the questionnaire response rate, a face to face questionnaire was carried out with nursing staff only. This method provided instant data, revealing $12.5 \%$ awareness and use of SQiD on that particular test day. Consequently it was felt that awareness raising was necessary before proceeding with the initial plan to test the incorporation of SQiD into the care round form.

See supplementary file: ds4130.docx - "PDSA 1 Graph"

\section{Design}

It was predicted that awareness of SQiD would be approximately $80 \%$ since this was the proportion of nursing staff that had attended training on the early recognition and management of delirium, in which SQiD was promoted. However, data revealed only $12.5 \%$ awareness and use of SQiD. At this stage (week four of the eight week project) it was decided to postpone the original plan of incorporating SQiD into the care rounds as it was apparent further awareness raising was necessary. Therefore it was agreed, after discussion with ward staff, that a tangible prompt such as a poster or card would be tested to determine if awareness increased. Using the information and design promoted in the delirium training session, a poster as a visual prompt and a small business style card were created. A benefit of these interventions is that they remain in place beyond the duration of the project.

Design of the final intervention, incorporation of the SQiD question into the care round form, was achieved simply by including the question on the form, making a fifth factor/question to be considered by the nurse for each patient.

\section{Strategy}

Adopting the Model for Improvement,[4] changes were tested using PDSA cycles (Plan, Do, Study, Act). Within each cycle three key questions are considered:

- "What are we trying to accomplish?" - this focuses on the aim of the test

- "How will we know that a change is an improvement?" - this focuses on measurement and finally

- "What changes can we make that will result in improvement?" this focuses on the changes necessary to improve results.

Measurement was important to determine if a change was an improvement. The outcome measure of this project was an increase in the percentage of patients over 75 upon which SQiD was used. Applying process measures (an increase in the percentages of staff who are aware of SQiD, the percentage of nurses using SQiD, and the percentage of times SQiD is documented as being asked on care round) was necessary to achieve the outcome measure.

Balancing measures were necessary to ensure that a change did not have a detrimental effect on any other area. In this case it was important to ensure that a change did not increase the perceived paperwork burden for staff. This could be done by making the change as simple as possible and incorporating it into current practice.

PDSA 1: Establish baseline of the awareness and current use of SQiD within ASRU. This involved development of a questionnaire which was distributed to 44 members of the MDT. Due to the response rate of our questionnaires (45\%), the unexpectedly low figures (predicted awareness $80 \%$ - actual $35 \%$ ) and the limit of an eight week timescale for the project, it was decided to reduce the scale and scope of the project by limiting the questionnaire to the nursing team only, instead of the MDT.

PDSA 2: Measure the percentage of nursing staff's awareness and use of SQiD. In an effort to improve the reliability and speed of gathering baseline awareness data, a face to face questionnaire was considered more effective. Applying this method provided instant results compared to the previous questionnaire, which took three weeks in total. Analysis of this data revealed 12.5\% awareness and use of SQiD, highlighting the need for an additional prompt or reminder of SQiD to promote awareness. A small information card and poster was developed, which provided pertinent information as to the definition of SQiD, the question itself ("Is this person more confused than before?") and the predisposing factors of delirium in acronym form. It was planned to distribute these cards to nursing team members and display posters around the ward to increase awareness.

PDSA 3: Measure the effectiveness of tangible prompts for nursing staff to increase awareness and use of SQiD. The concept was introduced to nursing staff at the early morning handover one day, explaining the reasons for the effort. Each nurse was then provided with a SQiD information card. Posters were displayed on each nursing bay and at key positions around the ward. Data were gathered at the end of shift to determine if the card had improved awareness of SQiD and if SQiD had been used in practice that day. Analysis of the data revealed feedback was received from only six of the nine staff due to the busy ward environment that day. Of the six staff involved, awareness had increased to $83 \%$ and use to $20 \%$ during this test. With nursing awareness at a sufficient level it was decided to test the SQiD question incorporated into the care round form in the next PDSA cycle.

PDSA 4: Test if incorporating SQiD into the care round form increases the awareness and use of SQiD. This was tested on a nurse caring for one over 75 year old patient for the duration of one shift. It was anticipated that the care round form would act as a prompt to apply SQiD to practice and a document from which to 
measure results. The staff member reported that the SQiD question was simple to use and that having it as a prompt on the care round form ensured its use throughout the shift. Study of the data indicated $100 \%$ awareness and $100 \%$ use. It was agreed that the scale of the test would be increased to three nursing staff members for the final PDSA cycle.

PDSA 5: Test if incorporating SQiD into the care round form increases the awareness and use of SQiD. Although the test sample was increased to three, on the day of the test one nurse was unable to carry out the test due to the burden of work. Study of the data indicated $100 \%$ awareness and $50 \%$ use during this test cycle.

This was the final cycle in our project as we considered the next step to be implementing the change by incorporating SQiD into the care round form for the whole ward which involved input from management.

\section{Results}

Baseline data revealed awareness of SQiD within the MDT as 35\% and use of SQiD as $15 \%$. Due to the recent delivery of delirium awareness training, this figure was predicted to be much higher. It was apparent, due to limitations of timescale and experience, that the scale and scope of the project should narrow and focus particularly on nursing staff. Once again, analysis of the data revealed a lesser than predicted awareness and use of SQiD (12.5\% awareness and use) despite $80 \%$ of nursing staff have attended delirium training at which SQiD was briefly introduced.

Following awareness raising activities, which included introduction of SQiD at nursing staff handover meeting, card distribution and poster display, a face to face survey indicated an increase to $83 \%$ awareness and $20 \%$ usage of SQiD. Although $100 \%$ awareness and use was anticipated at this stage, the busy ward environment prevented $33 \%$ of nursing staff responding during the test.

PDSA 4 and 5 involved the incorporation the SQID question into the care rounds which resulted in $100 \%$ awareness and $50 \%$ use during the test. This shows an increase in both awareness and use however it was a small scale test. Sustainability of this improvement required further implementation of the newly introduced care rounds.

A face to face survey was used to measure how easy SQiD was for the staff to use. Both quantitative and qualitative data suggested SQiD was easily incorporated into practice. A further measure for consideration was the potential for a percentage of patients to falsely screen positive when SQiD was applied. This was not reported during our test.

See supplementary file: ds4069.docx - "\% staff awareness/use run charts"

\section{Lessons and limitations}

Despite delirium awareness training being delivered to the majority of staff the link between SQiD and delirium did not appear to be clear. Our data revealed that awareness was not at the anticipated level of $80 \%$. This indicates that training does not always ensure knowledge and understanding, and certainly not implementation.

Due to the short timescale, the benefit of using data collection methods that provided instant data was recognised early on in the project. Additionally, recognising the importance of gathering the correct data was key learning. In selecting a questionnaire to collate baseline data that could be used pre and post intervention, the skill required in its development was perhaps underestimated. Care was required to ensure that the questions elicited the type of answer that could be counted quantitatively or qualitatively. Several drafts were made and tested with a willing member of staff before general distribution. Response was poorer and took much longer than expected, leading us to review our strategy for data collection during the project. The initial questionnaire yielded a $45 \%$ response rate whilst face to face surveys provided instant and higher response rates.

There were many reflective learning points associated with this project, including the importance of investing in staff engagement and the need for frequent project reports to maintain enthusiasm and momentum for the project. Designation of an identified leader from the clinical team may have encouraged more general staff engagement, resulting in the project progressing further in the allocated time.

The project focus was a national priority and because of this it was assumed that this project would be adopted more readily by staff. However, the introduction of another new initiative in the workplace made it difficult to engage staff initially. On reflection, it was felt that staff could have engaged with the work more readily if they had been consulted on what they would like to improve.

As nursing students undertaking an improvement project during an eight week clinical placement, it was challenging to ensure the project work was not detrimental to placement learning objectives. However, a factor which promoted success in this area was the support provided by faculty and healthcare sponsor team.

Utilising Deming's lens of profound knowledge would have been a useful tool to have used for this project, to enable understanding and appreciation of the system that we worked within, and the psychology behind staff thinking.[4] This tool will be used to inform future improvement projects, which should ensure greater success.

Resilience and self-efficacy are important personal factors which have developed throughout this improvement project journey. Additionally, understanding and appreciating the importance effective communication has in the success of improvement work has been key learning.

\section{Conclusion}

Following awareness raising activities, the awareness and use of SQiD increased from $12.5 \%$ to $83 \%$ and $20 \%$ respectively. 


\section{BMJ Quality Improvement Reports}

Incorporating the SQiD question into the care round form delivered $100 \%$ awareness and $50 \%$ use during testing. Although this small scale project could be viewed as a success, the requirements for sustainability depend upon further implementation and spreading of the change. Sustained improvement is also dependent upon the successful implementation of the care round forms which were only recently adopted.

Undertaking this improvement project has provided valuable learning in both quality improvement science and personal learning. The improved knowledge and understanding of effective communication and the intricacies of team working is transferrable and will benefit future nursing practice.

\section{References}

1. National Institute for Health and Care Excellence [Internet]. UK: Delirium: diagnosis, prevention and management. NICE 2010. [cited 2014 Sep 27] Available from:

http://www.nice.org.uk/guidance/cg103/chapter/introduction

2. Reston JT, and Schoelles KM. In-facility delirium prevention programs as a patient safety strategy: a systematic review. Ann Int Med 2013; 158(5 part 2): 375-80.

3. Sands MB, et al. Single question in delirium (SQiD): testing its efficacy against psychiatrist interview, the Confusion Assessment Method and Memorial Delirium Assessment Scale. Palliative Med 2010;24(6):561-5.

4. Langley GL, Moen R, Nolan KM, Nolan TW, Norman CL, Provost LP. The Improvement Guide: A Practical Approach to Enhancing Organizational Performance (2nd Edition). San Francisco, California, USA: Jossey-Bass Publishers; 2009.

\section{Declaration of interests}

Nothing to declare.

\section{Acknowledgements}

Dr Eloise Pearson, Dr Sandra Hainey, Marie Hanlin, and Tayside Centre for Organisational Effectiveness QI team, Ward 7 ASRU staff, Ninewells Hospital. 